\title{
Tension Pyopneumothorax due to a Ruptured Pulmonary Echinococcal Cyst
}

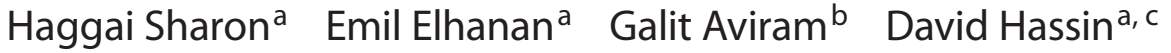 \\ ${ }^{\mathrm{a}}$ Department of Internal Medicine $\mathrm{H}^{\mathrm{b}}{ }^{\mathrm{b}}$ Imaging Department, and ${ }^{\mathrm{C}}$ Infectious Diseases Unit, Tel Aviv Sourasky \\ Medical Center, Tel Aviv, Israel
}

A 38-year-old otherwise healthy man presented to the emergency department with a 3-day history of fever, dyspnea, pleuritic chest pain, and hemoptysis. CT scan suggested a hydatid lung cyst (fig. 1) without evidence of other organ involvement. Sputum microscopy revealed echinococcal protoscolices of various maturations, and sputum cultures yielded Acinetobacter baumanii. The patient refused surgery and was discharged for medical therapy with 2 weeks of oral clindamycin and levofloxa- cin as well as 6 months of daily albendazole $400 \mathrm{mg}$ b.i.d. for the first 3 weeks of every month. A week later he was readmitted due to recurrent syncope and hypotension. He had an elevated JVP and no breath sounds on the right side. There was also marked new eosinophilia. CT demonstrated a new massive right pleural effusion compressing the superior vena cava and right heart and necessitating emergency drainage of what was an overt empyema with a $\mathrm{pH}$ of 6.8 (fig. 2a). During hospitalization he re-

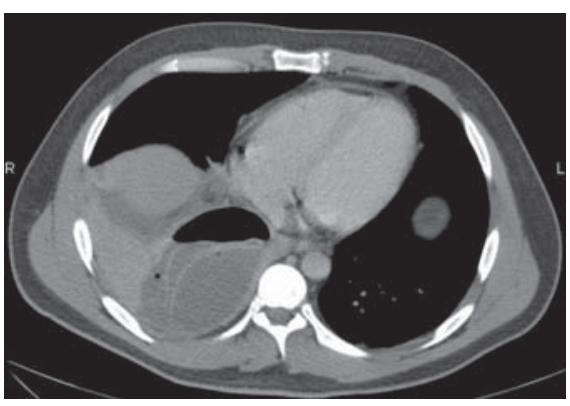

Fig. 1. CT scan suggesting a hydatid lung cyst.
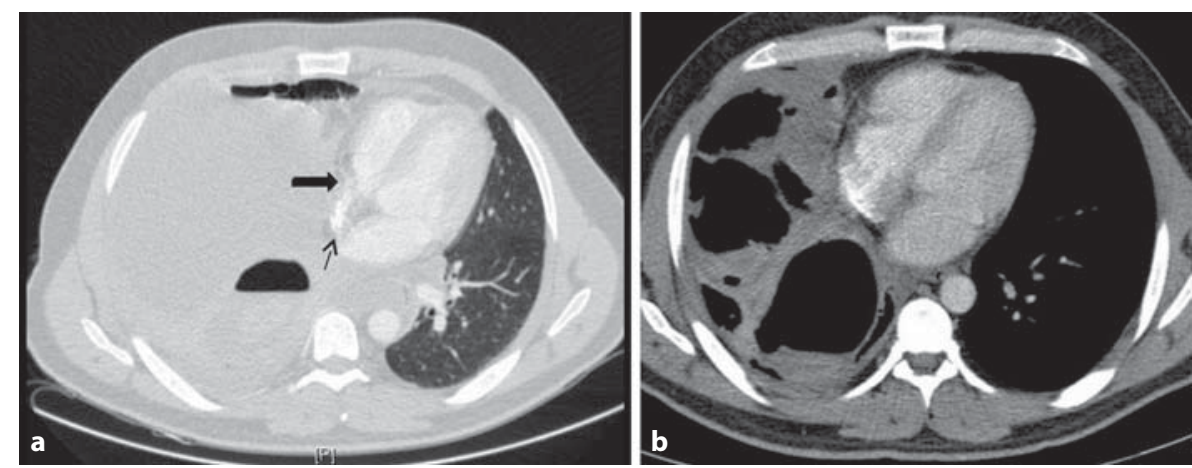

Fig. 2. a Parenchymal window depicting free air and fluid in the chest cavity. Note the leftward shift of the mediastinum. Also note the thick arrow indicating marked indentation of the right atrium and the thin arrow indicating obliteration of the SVC (starting higher in the mediastinum) by marking the position where the missing radiopaque shadow of the SVC entering the right atrium can usually be seen. $\mathbf{b}$ Two years later, the cyst seemed empty with surrounding pleuropulmonary fibrosis.

\section{KARGER}

Fax +4161306 1234

E-Mail karger@karger.ch

www.karger.com
(C) 2012 S. Karger AG, Basel

0025-7931/12/0844-0327\$38.00/0

Accessible online at:

www.karger.com/res
Dr. Haggai Sharon

Internal Medicine H, Tel Aviv Sourasky Medical Center 6 Weitzman St.

Tel Aviv 64239 (Israel)

Tel. +972 36973 376, E-Mail haggais@tasmc.health.org.il 
fused definitive surgery again and so was treated only with IV piperacillin/tazobactam and clindamycin as well as with his previously prescribed oral albendazole regimen. He was then released for a further 5 months of oral albendazole therapy. Two years later, the cyst seemed empty with surrounding pleuropulmonary fibrosis (fig. 2b).

Lung involvement occurs in 10-30\% of cases of cystic echinococcosis. Most patients remain asymptomatic for years, with $50 \%$ presenting with complications on initial diagnosis [1]. In patients from endemic areas, therefore, hydatid disease should be included in the differential diagnosis of a one-sided pleural effusion even years after migration. Principal complications are cyst rupture causing pneumothorax, pleural effusion, empyema with secondary seeding, and superimposed infection manifesting as a pulmonary abscess [2], as in this patient. Two rapidly fatal complications are anaphylactic shock or tension pyopneumothorax. Treatment is surgery with subsequent chemotherapy [3]. Long courses of albendazole or albendazole with praziquantel are usually given when surgery is not available. Although response to medical therapy alone is usually poor in large lesions, it may sometimes result in a good clinical outcome [3], as was the case in our patient.

References

Santivanez S, Garcia HH: Pulmonary cystic echinococcosis. Curr Opin Pulm Med 2010; 16:257-261.

-2 Bagheri R, et al: Pulmonary hydatid cyst: analysis of 1,024 cases. Gen Thorac Cardiovasc Surg 2011;59:105-109.
Dziri C, Haouet K, Fingerhut A, Zaouche A: Management of cystic echinococcosis complications and dissemination: where is the evidence? World J Surg 2009;33:1266-1273. 